\title{
Differences in postprandial inflammatory responses to a 'modern' v. traditional meat meal: a preliminary study
}

\author{
Fatemeh Arya ${ }^{1}$, Sam Egger ${ }^{2}$, David Colquhoun ${ }^{3}$, David Sullivan ${ }^{4},{\text { Sebely } \text { Pal }^{5} \text { and Garry Egger }}^{6 *}$ \\ ${ }^{1}$ School of Human Nutrition, University of Sydney, Sydney, NSW, Australia \\ ${ }^{2}$ Cancer Council NSW, Sydney, NSW, Australia \\ ${ }^{3}$ University of Queensland, Brisbane, QLD, Australia \\ ${ }^{4}$ Royal Prince Alfred Hospital, Sydney, NSW, Australia \\ ${ }^{5}$ School of Public Health; ATN Centre for Metabolic Fitness, Curtin University, Perth, WA, Australia \\ ${ }^{6}$ School of Health and Applied Sciences, Southern Cross University, Lismore, NSW, Australia \\ (Received 2 November 2009 - Revised 1 March 2010 - Accepted 2 March 2010 - First published online 9 April 2010)
}

\begin{abstract}
A low-grade inflammatory response ('metaflammation') has been found to be associated with certain chronic diseases. Proposed inducers of this have been aspects of the modern lifestyle, including newly introduced foods. Plasma TAG, and the inflammatory cytokines C-reactive protein (CRP), TNF- $\alpha$ and IL-6 were compared in a randomised, cross-over trial using ten healthy subjects before and after eating $100 \mathrm{~g}$ of kangaroo, or a 'new' form of hybridised beef (wagyu) separated by about 1 week. Postprandial levels for 1 and $2 \mathrm{~h}$ of TAG, IL- 6 and TNF- $\alpha$ were significantly higher after eating wagyu compared with kangaroo ( $P=0.002$ for TAG at $1 \mathrm{~h}, P<0.001$ at $2 \mathrm{~h} ; P<0.001$ for IL-6 and TNF- $\alpha$ at 1 and $2 \mathrm{~h}$ ). CRP was significantly higher $1 \mathrm{~h}$ postprandially after wagyu $(P=0.011)$ and non-significantly higher $2 \mathrm{~h}$ postprandially $(P=0.090)$. We conclude that the metaflammatory reaction to ingestion of a 'new' form of hybridised beef (wagyu) is indicative of a low-grade, systemic, immune reaction when compared with lean game meat (kangaroo). Further studies using isoenergetic intake and isolating fatty acid components of meats are proposed.
\end{abstract}

Kangaroo meat: Wagyu meat: Environment: Cross-over studies: Evolution

There is evidence of a link between a form of low-grade systemic inflammation and several chronic diseases ${ }^{(1,2)}$. This subclass of inflammation has been labelled 'metaflammation' (metabolically triggered inflammation) ${ }^{(3)}$, 'paraflammation' ${ }^{(4)}$, or 'smouldering inflammation' ${ }^{(5)}$. It does not include the features of classical microbial-related inflammation (pain, redness, swelling and fever), and is characterised only by a mild increase in plasma levels of certain pro-inflammatory markers such TNF- $\alpha$, IL-6 and C-reactive protein (CRP). Obesity is known to be associated with this form of inflammation $^{(2)}$. However, a number of other "inducers' ${ }^{(4)}$ have also been causally implicated, some with, but some without obesity $^{(6)}$. This has led to the suggestion that certain lifestyle-related stimuli, including 'new' foods with which humans have not evolved, have a pro-inflammatory effect, while stimuli with which humans have a long history have an anti-inflammatory, or neutral effect ${ }^{(7)}$.

Limited studies have shown that lean meat is less likely to raise oxidative stress or cause inflammation than fatty meat ${ }^{(8)}$. To our knowledge, however, no research to date has compared the effects of fatty $v$. lean meat meals on inflammatory markers within the same individual. The former type of meat is more characteristic of modern domesticated cattle, whereas the latter is more common in free-range and game animals. We hypothesised that eating a type of fatty meat with which humans have not had a long nutritional history may stimulate a mild-level immune reaction, and acute inflammatory response, when compared with the ingestion of a form of lean game meat, with which evolving humans have been more familiar. We tested this in a cross-over trial by comparing postprandial inflammatory markers and plasma TAG after ingestion of kangaroo and wagyu beef. Kangaroo is part of a traditional Aboriginal diet in Australia. It contains $<4 \%$ fat on average, with $<1 \%$ of this saturated. Wagyu, a newly hybridised form of domestic cattle, was chosen as an example of modern meat, relatively high in fat $(25-30 \%)$, of which about $40 \%$ is saturated.

\section{Methods \\ Participants}

Ten healthy subjects (six males, four females), aged $26 \cdot 5$ (range 19-38) years, BMI 21.6 (range $18-26.5$ ) $\mathrm{kg} / \mathrm{m}^{2}$ with no history of metabolic disease or current medication use, and with average physical activity levels (i.e. not participating 
in training activities) were recruited to examine postprandial inflammatory markers on two separate occasions.

\section{Procedure}

The present study used a randomised cross-over design with longitudinal components. Participants were fasted for $10 \mathrm{~h}$ before each testing session. Loin steak $(100 \mathrm{~g})$ from either kangaroo or wagyu was given with a standardised portion of baked potato $(75 \mathrm{~g})$ and green peas $(50 \mathrm{~g})$. The meals were not intended to be isoenergetic. Five participants were randomly selected to be given either kangaroo or wagyu at their first sitting. They were then crossed over to the other meal at their second sitting, 6-10d later. Meat was pan-cooked, with no added oil or salt, and visible fat was trimmed from the wagyu before cooking. Subjects were confined, without food or drink, during postprandial measurements. Fig. 1 shows the study design.

Blood samples were taken immediately before each test meal (baseline) and hourly for $2 \mathrm{~h}$ after each meal given at approximately 08.00 hours on two separate days, separated by a 'wash-out' period of approximately 1 week (maximum of $10 \mathrm{~d}$, minimum of $6 \mathrm{~d}$ ). Blood was drawn according to standard guidelines. Serum was separated from fresh samples by centrifuging. Serum samples were stored in a ' $-70^{\circ} \mathrm{C}$ ' freezer until being transported for analysis.

\section{Outcome measures}

Plasma IL-6, TNF- $\alpha$ and CRP levels were measured by a solid-phase enzyme amplified sensitivity immunoassay performed on microtiterplates according to the manufacturers' instructions (TNF- $\alpha$ EASIA and IL-6 EASIA kits, BioSource, Nivelles, Belgium; CRP, Alpha Diagnostics International, San Antonio, TX, USA). Samples were measured at a wavelength of $450 \mathrm{~nm}$ against a reference filter set at $630 \mathrm{~nm}$ using a Bio-Rad 550 microplate reader (Bio-Rad, Hercules, CA, USA). Other assays, measured through standard means were TAG, total and HDL-cholesterol, blood glucose and creatine. Of these, only TAG is reported here. A total of sixty assay sets $(2$ meals $\times(1$ baseline +2 postprandial $) \times 10$ subjects) were done.

\section{Statistical analysis}

Regression analyses with generalised estimating equation adjustment for repeated observations on the same individual were used to examine (a) baseline-adjusted effects of meal type (kangaroo $v$. wagyu) on each of the four outcomes at 1 and $2 \mathrm{~h}$ and (b) changes in outcomes from baseline values associated with each meal type. Natural log transformations were performed on each of the four outcome variables to encourage Gaussian distributions and the transformed outcomes were used in all regression analyses. As a consequence of using log-transformed outcomes, effects are reported in terms of proportionate changes in means rather than mean differences. Four primary regression models corresponding to the four outcomes were used to examine the above study objectives, with each model sharing a common formulation. Categorical covariates included meal type (kangaroo and wagyu), time after meal (0,1 and $2 \mathrm{~h}$ ) and meal sitting (first and second sittings). In addition, a term for the interaction between meal type and time after meal was included, regardless of its statistical significance, to allow estimation of separate meal effects at 1 and $2 \mathrm{~h}$. The lone continuous covariate included in each model was the natural log of the outcome baseline value. Exchangeable working correlation structures were used for the primary analysis, with sensitivity analyses being performed using various other working correlation matrices (these results are not reported because differences were negligible). Model-based variance estimators were used in all regression models. To assess the evidence of carry-over effects, each of the four primary models was refitted to the data with the inclusion of an additional interaction term for meal type by meal sitting. However, because these terms did not indicate evidence of carry-over effects for any of the outcomes, they were not included in the primary analysis models.

The present study was conducted according to the guidelines laid down in the Declaration of Helsinki and all procedures involving human subjects were approved by the University if Sydney ethics committee. Written informed consent was obtained from all subjects.

\section{Results}

Figs. 2(a)-(d) show the mean response trajectories for (natural log) TAG, IL-6, TNF- $\alpha$ and CRP, respectively.

Estimates of selected effects of interest on each of the four outcomes are shown in Table 1.

Table 1 shows that wagyu was associated with significant postprandial increases in each of the four outcome measures (ranging from $P=0.022$ to $P<0.001$ ). This increase was most extreme in the TNF- $\alpha$ measure which increased by
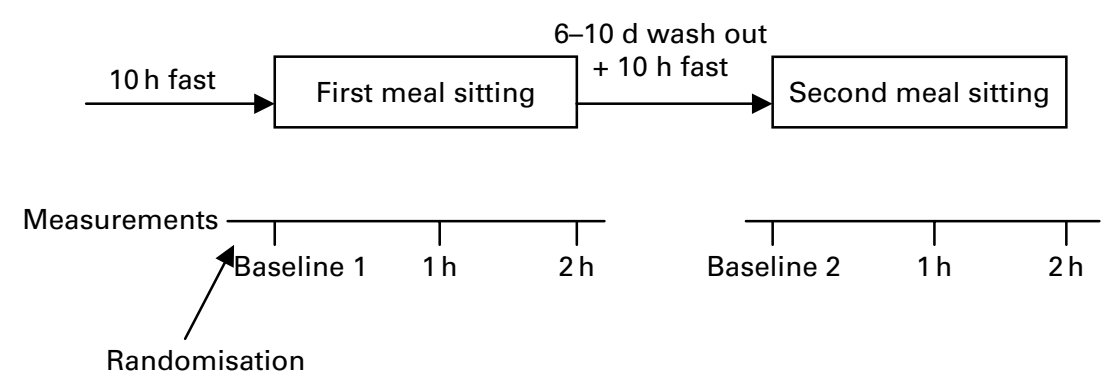

Fig. 1. Cross-over design of the study. 

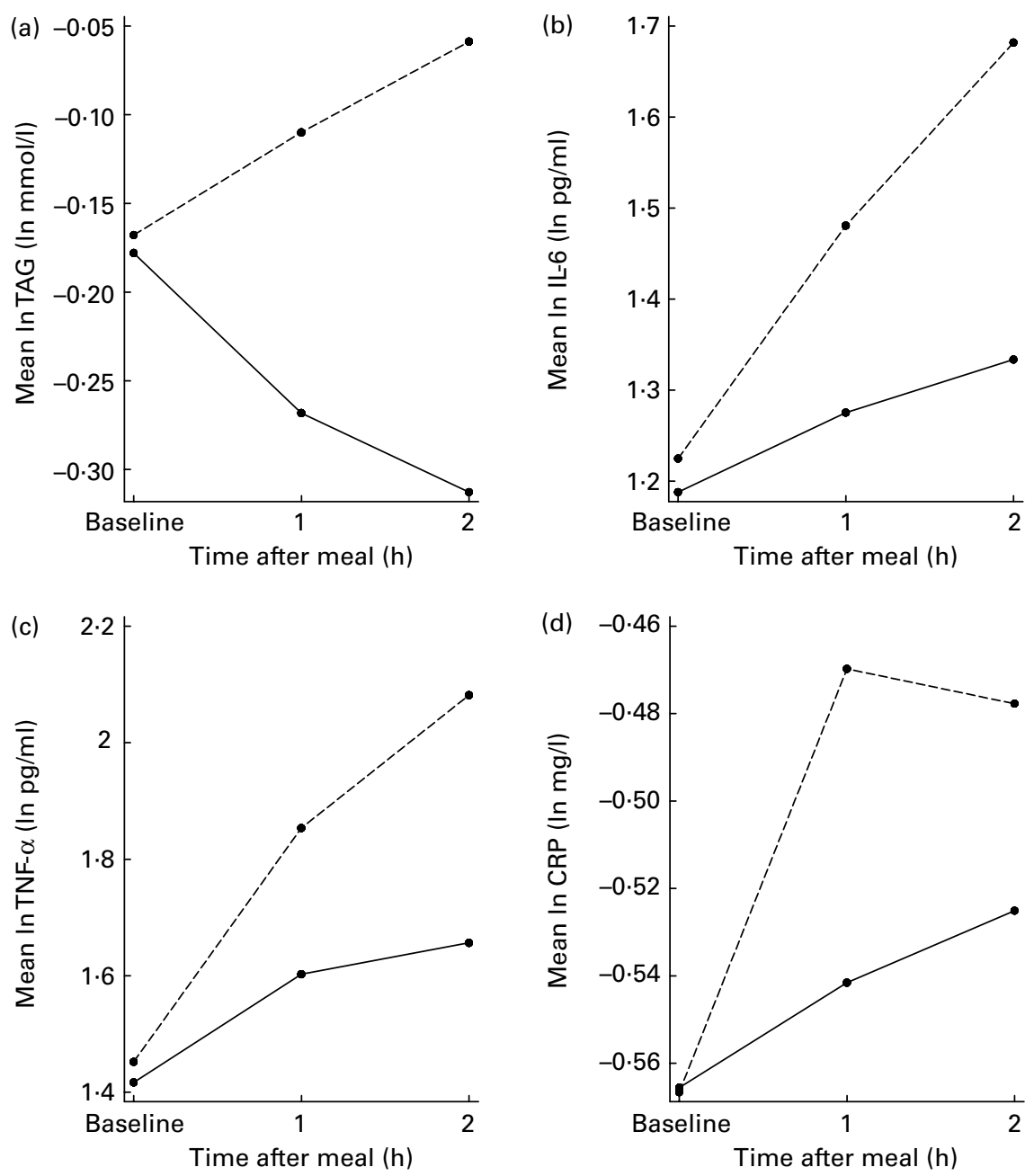

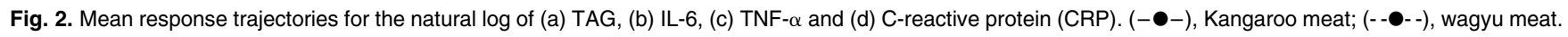

$78(95 \%$ CI 50,111$) \%$ at $2 \mathrm{~h}$ postprandial. Kangaroo was found to be associated with significant increases in IL-6 and TNF- $\alpha$ counts $(P<0.001$ for both measures $)$ and a non-significant increase in CRP. Interestingly, a significant declining trend in postprandial TAG was found to be associated with kangaroo $(P=0.005)$. Specifically, declines from baseline values were recorded in six of the ten participants' TAG measures at 1 and $2 \mathrm{~h}$ after kangaroo consumption. At $1 \mathrm{~h}$ postprandial, wagyu was associated with a 16 (95\% CI 6, 28) $\%$ higher TAG count, a $19(95 \%$ CI 10, 28) \% higher IL-6 count, a 26 (95\% CI 12, 42) \% higher TNF- $\alpha$ count and an 8 (95\% CI 2,14) \% higher CRP count compared with kangaroo (Table 1). At $2 \mathrm{~h}$, wagyu was associated with a $28(95 \%$ CI 16, 40) \% higher TAG count, a $37(95 \%$ CI $27,48) \%$ higher IL-6 count and a 50 (95\% CI 33, 70) \% higher TNF- $\alpha$ count compared with kangaroo. Blood counts of CRP were non-significantly higher $2 \mathrm{~h}$ after eating wagyu $(P=0.090)$ compared with kangaroo.

\section{Discussion}

Low-grade systemic inflammation ('metaflammation') has been proposed as an underlying cause of much chronic disease ${ }^{(3)}$. Several 'inducers' of this inflammatory reaction have recently been elucidated ${ }^{(6,9,10)}$, with the suggestion that these fall into a category of lifestyle and environmental stimulants that are relatively new to humans, probably originating as a function of the modern techno-industrial environment ${ }^{(6)}$. Our findings show that a 'newly' introduced form of beef (wagyu) has a significantly greater postprandial inflammatory effect than a traditional kind of meat (kangaroo).

Whilst kangaroo is native to Australia, it was used here as an example of lean game meats eaten by humans over millennia. Wagyu beef, on the other hand, is at the opposite end of the spectrum in terms of human adaptation, being hybridised only in the last two to three decades. Wagyu is by no means the fattiest of meats available in the modern market place, and has a high proportion of MUFA and $n-3$ fatty acids relative to other beef. As visible fat was also cut from the meat in the present study, the difference in inflammatory reaction may have been even greater between kangaroo and other beef.

Our suggestion of a difference in the physiological response to modern $v$. historically consumed foods (i.e. foods with which humans have clearly adapted) is not new ${ }^{(11)}$. However, it expands existing anthropological data to include the effects of lifestyle-related inflammatory inducers of chronic disease ${ }^{(12)}$. While long-term health effects cannot be presumed from these findings, the change in inflammatory indices is indicative 
Table 1. Potential predictors of change in TAG, IL-6, TNF- $\alpha$ and C-reactive protein (CRP)

(Relative difference percentages and $95 \%$ confidence intervals)

\begin{tabular}{|c|c|c|c|c|c|c|c|c|c|c|c|c|c|c|c|c|}
\hline \multirow[b]{3}{*}{ Predictor } & \multicolumn{4}{|c|}{ TAG } & \multicolumn{4}{|c|}{ IL-6 } & \multicolumn{4}{|c|}{ TNF- $\alpha$} & \multicolumn{4}{|c|}{ CRP } \\
\hline & \multicolumn{2}{|c|}{ Relative difference } & \multirow[b]{2}{*}{$P$} & \multirow[b]{2}{*}{$P$ trend } & \multicolumn{2}{|c|}{ Relative difference } & \multirow[b]{2}{*}{$P$} & \multirow[b]{2}{*}{$P$ trend } & \multicolumn{2}{|c|}{ Relative difference } & \multirow[b]{2}{*}{$P$} & \multirow[b]{2}{*}{$P$ trend } & \multicolumn{2}{|c|}{ Relative difference } & \multirow[b]{2}{*}{$P$} & \multirow[b]{2}{*}{$P$ trend } \\
\hline & $\%$ & $95 \% \mathrm{Cl}$ & & & $\%$ & $95 \% \mathrm{Cl}$ & & & $\%$ & $95 \% \mathrm{Cl}$ & & & $\%$ & $95 \% \mathrm{Cl}$ & & \\
\hline \multicolumn{17}{|l|}{ Meal } \\
\hline \multicolumn{17}{|l|}{ At $1 \mathrm{~h}$ after meal ${ }^{*}$} \\
\hline Kangaroo & \multicolumn{2}{|c|}{ Reference } & & & \multicolumn{2}{|c|}{ Reference } & & & \multicolumn{2}{|c|}{ Reference } & & & \multicolumn{2}{|c|}{ Reference } & & \\
\hline Wagyu & 16 & 6,28 & 0.002 & & 19 & 10,28 & $<0.001$ & & 26 & 12,42 & $<0.001$ & & 8 & 2,14 & 0.011 & \\
\hline \multicolumn{17}{|l|}{ At $2 \mathrm{~h}$ after meal ${ }^{\star}$} \\
\hline Kangaroo & \multicolumn{2}{|c|}{ Reference } & & & \multicolumn{2}{|c|}{ Reference } & & & \multicolumn{2}{|c|}{ Reference } & & & & rence & & \\
\hline Wagyu & 28 & 16,40 & $<0.001$ & & 37 & 27,48 & $<0.001$ & & 50 & 33,70 & $<0.001$ & & 5 & $-1,11$ & 0.090 & \\
\hline Time after meal & & & & & & & & & & & & & & & & \\
\hline Kangaroo* $^{\star}$ & & & & & & & & & & & & & & & & \\
\hline Baseline & & erence & & & & ence & & & & ence & & & & rence & & \\
\hline $1 \mathrm{~h}$ & -9 & $-17,0$ & 0.062 & 0.005 & 9 & 1,18 & 0.022 & $<0.001$ & 20 & 7,36 & 0.003 & $<0.001$ & 2 & $-3,8$ & 0.400 & 0.168 \\
\hline $2 \mathrm{~h}$ & -13 & $-21,-4$ & 0.005 & & 16 & 7,25 & $<0.001$ & & 27 & 13,43 & $<0.001$ & & 4 & $-2,10$ & 0.156 & \\
\hline Wagyu* & & & & & & & & & & & & & & & & \\
\hline Baseline & & erence & & & & ence & & & & ence & & & & rence & & \\
\hline $1 \mathrm{~h}$ & 6 & $-4,16$ & 0.231 & 0.022 & 29 & 20,39 & $<0.001$ & $<0.001$ & 49 & 32,69 & $<0.001$ & $<0.001$ & 10 & 4,17 & $<0.001$ & 0.002 \\
\hline $2 \mathrm{~h}$ & 17 & 2,33 & 0.024 & & 49 & 34,66 & $<0.001$ & & 78 & 50,111 & $<0.001$ & & 8 & $-1,16$ & 0.073 & \\
\hline Period $^{\star}$ & & & & & & & & & & & & & & & & \\
\hline First sitting & & erence & & & & ence & & & & ence & & & & rence & & \\
\hline Second sitting & 1 & $-5,6$ & 0.821 & & -4 & $-8,0$ & 0.066 & & 1 & $-5,9$ & 0.687 & & -3 & $-6,0$ & 0.057 & \\
\hline $\begin{array}{l}\text { Log baseline } \\
\text { (per unit increase })^{\star}\end{array}$ & 154 & 134,175 & $<0.001$ & & 144 & 109,186 & $<0.001$ & & 71 & 46,100 & $<0.001$ & & 163 & 146,181 & $<0.001$ & \\
\hline $\begin{array}{l}\text { Treatment } \times \text { period } \\
\text { interactiont }\end{array}$ & & & & & & & & & & & & & & & & \\
\hline Meal effects & & & & & & & & & & & & & & & & \\
\hline First sitting & & erence & & & & ence & & & & ence & & & & rence & & \\
\hline Second sitting & 1 & $-12,15$ & 0.914 & & -12 & $-23,1$ & 0.072 & & 4 & $-6,15$ & 0.439 & & 5 & $-8,19$ & 0.470 & \\
\hline
\end{tabular}

${ }^{*}$ Covariates included in the primary regression models.

$\dagger$ Evidence of carry-over effects was assessed by adding terms for the interaction of treatment (meal) $\times$ period (meal sitting) to each of the four primary regression models. 
of health benefits of different forms of foods, which needs to be investigated further. We have not speculated here about the cause of such an effect beyond the 'natural' conditions of both forms of meat. The rationale of the study was to compare, in a preliminary fashion, whole foods rather than individual ingredients, such as fat content or type, on the human immune response, as is recommended in such studies ${ }^{(13)}$. We can make no judgements about specific causes on the basis of these findings. Further work now needs to be done comparing isoenergetic effects and specific components of the different meats, as well as possible comparisons between other 'old' and 'modern' whole meats. Previous findings of the potent inflammatory effects of saturated fats ${ }^{(14)}$ suggest this as a hypothesis for further testing. It would also be of interest to measure inflammatory effects within free-range $v$. domesticated animals themselves, in line with the principle that 'we are not just what we eat, but what we eat, eats'(15), and to look at other inflammatory and atherogenic indices such as flow-mediated diameter.

\section{Acknowledgements}

No grant funding was received for this research.

F. A. was the student researcher; S. E. was the bio-medical statistician; D. C., D. S. and S. B. were consultants; G. E. was the supervisor and senior author.

There are no conflicts of interest.

\section{References}

1. Arner $P$ (2007) Introduction: the inflammation orchestra in adipose tissue. J Intern Med 262, 404-407.
2. Bulló M, Casas-Agustench P, Amigó-Correig P, et al. (2007) Inflammation, obesity and comorbidities: the role of diet. Public Health Nutr 10, 1164-1172.

3. Hotamisligil GS (2006) Inflammation and metabolic disorders. Nature 444, 860-867.

4. Medzhitov R (2008) Origin and physiological roles of inflammation. Nature 454, 428-435.

5. Mantovani A, Allovena P, Sica A, et al. (2008) Cancer related inflammation. Nature 454, 436-444.

6. Egger G \& Dixon J (2009) Obesity and chronic disease: always offender, or often just accomplice? Br J Nutr 102, 1238-1242.

7. Egger G \& Dixon J (2009) Should obesity be the main game? Or do we need an environmental makeover to combat the inflammatory and chronic disease epidemics? Obes Rev 10, 237-249.

8. Hodgson JM, Ward N, Burke V, et al. (2007) Increased lean meat intake does not elevate markers of oxidative stress and inflammation in humans. J Nutr 137, 363-368.

9. Lamon BD \& Hajar DP (2008) Inflammation at the molecular interface of atherogenesis: an anthropological journey. Am J Pathol 173, 1253-1264.

10. Warnberg J, Nova E, Romeo J, et al. (2007) Lifestyle-related determinants of inflammation in adolescence. Br J Nutr 98, S116-S120.

11. Cordain L, Eaton SB, Brand-Miller J, et al. (2002) The paradoxical nature of hunter-gatherer diets: meat-based, yet nonatherogenic. Eur J Clin Nutr 56, S42-S52.

12. O'Keefe JH \& Bell DS (2007) Postprandial hyperglycemia/ hyperlipidemia (postprandial dysmetabolism) is a cardiovascular risk factor. Am J Cardiol 100, 899-904.

13. Jacobs DR \& Tapsell LC (2007) Food, not nutrients, is the fundamental unit in nutrition. Nutr Rev 65, 439-450.

14. Margioris A (2009) Fatty acids and postprandial inflammation. Curr Opin Clin Nutr Metab Care 12, 129-137.

15. Roberts P (2008) The End of Food. London: Bloomsbury Publishing. 